\title{
Special Issue on Algorithms and Data Structures (WADS 2019)
}

\author{
Zachary Friggstad $^{1}$ · Jörg-Rüdiger Sack ${ }^{2} \cdot$ Mohammad R. Salavatipour $^{1}$
}

Published online: 3 February 2021

(C) The Author(s), under exclusive licence to Springer Science+Business Media, LLC part of Springer Nature 2021

This special issue contains selected papers from those presented at the 16th International Algorithms and Data Structures Symposium (WADS 2019), which was held from August 5 to August 7, 2019, in Edmonton, Alberta, Canada. WADS, which alternates with the Scandinavian Symposium and Workshops on Algorithm Theory, SWAT, is a forum for researchers in the area of design and analysis of algorithms and data structures.

In response to the call for papers, 88 papers were submitted. From these submissions, the Program Committee carefully selected 42 papers for presentation at WADS 2019, using online discussion in EasyChair. From these 42, the Program Committee selected five outstanding algorithms papers for a special issue in Algorithmica. Extended versions of these papers underwent a rigorous refereeing process. They are presented here:

- Succinct Encodings for Families of Interval Graphs, by Hüseyin Acan, Sankardeep Chakraborty, Seungbum Jo and Srinivasa Rao Satti

- Graph Isomorphism for (H1, H2)-Free Graphs: An Almost Complete Dichotomy, by Marthe Bonamy, Nicolas Bousquet, Konrad K. Dabrowski, Matthew Johnson, Daniël Paulusma, and Théo Pierron

- Online bin covering with advice, by Joan Boyar, Lene M. Favrholdt, Shahin Kamali and Kim S. Larsen

- Guess Free Maximization of Submodular and Linear Sums, by Moran Feldman

- Improved Streaming Algorithms for Maximizing Monotone Submodular Functions under a Knapsack Constraint by by Chien-Chung Huang and Naonori Kakimura

We gratefully acknowledge the support of the WADS 2019 sponsors: University of Alberta, Pacific Institute for the Mathematical Sciences, Elsevier, and Springer.

Publisher's Note Springer Nature remains neutral with regard to jurisdictional claims in published maps and institutional affiliations.

Jörg-Rüdiger Sack

sack@scs.carleton.ca

1 Department of Computing Science, University of Alberta, Edmonton, AB T6G 2S4, Canada

2 School of Computer Science, Carleton University, Ottawa, ON K1S 5B6, Canada 